\title{
THE
}

$1-15-1993$

\section{A Study of Low Temperature Heat Capacity Anomalies in Bimetallic Alloy Clusters using J-Walking Monte Carlo Methods}

\author{
Gustavo E. Lopez \\ University of Rhode Island \\ David L. Freeman \\ University of Rhode Island, dfreeman@uri.edu
}

Follow this and additional works at: https://digitalcommons.uri.edu/chm_facpubs

Terms of Use

All rights reserved under copyright.

\section{Citation/Publisher Attribution}

Lopez, G. E., \& Freeman, D. L. (1993). A Study of Low Temperature Heat Capacity Anomalies in Bimetallic Moy Clusters Using J Walking Monte Carlo Methods. Journal of Chemical Physics, 98(2), 1428-1435. doi: 10.1063/1.464307

Available at: http://dx.doi.org/10.1063/1.464307

This Article is brought to you for free and open access by the Chemistry at DigitalCommons@URI. It has been accepted for inclusion in Chemistry Faculty Publications by an authorized administrator of DigitalCommons@URI. For more information, please contact digitalcommons-group@uri.edu. 


\title{
A study of low temperature heat capacity anomalies in bimetallic alloy clusters using J-walking Monte Carlo methods
}

\author{
Gustavo E. Lopez ${ }^{\text {a) }}$ and David L. Freeman \\ Department of Chemistry, University of Rhode Island, Kingston, Rhode Island 02881
}

(Received 27 August 1992; accepted 28 September 1992)

\begin{abstract}
Heat capacities are calculated as a function of temperature for bimetallic clusters composed of six palladium and seven nickel atoms using Monte Carlo techniques both with and without J-walking. By applying a simulated annealing strategy, the minimum energy configurations at $0 \mathrm{~K}$ are identified for a series of interatomic interaction strengths.

A significant dependence of the spatial arrangement of atoms on the strength of the Pd-Ni interaction is observed. Calculations of the heat capacity as a function of temperature show the presence of a "melting" coexistence region similar to that observed in pure clusters. For the alloy clusters, low temperature heat capacity anomalies are observed arising from isomerizations that are reminiscent of order-disorder transitions known to occur in some bulk alloy materials. These low temperature heat capacity anomalies are observable only when the $\mathbf{J}$-walking algorithm is used.
\end{abstract}

\section{INTRODUCTION}

An active field of research in recent years has been the study of atomic and molecular clusters. Clusters are ubiquitous in nature and knowledge of their physical and chemical properties leads to an understanding of such divergent areas as nucleation and crystal growth, phase transitions, heterogeneous catalysis, and atmospheric phenomena. ${ }^{1}$ In this work we will focus on some of the properties of cluster materials that are of importance to heterogeneous catalysis. Many catalysts consist of clusters of metal atoms embedded in a support material like silica. These dispersed metal clusters make good catalysts because the largest fraction of their atoms reside on the surface. The resulting large surface to volume ratio of metal clusters is important to their enhanced catalytic activity. The metal clusters that make up catalytic materials can either be pure substances or alloys. Alloy clusters have proved to be particularly valuable as catalysts ${ }^{2}$ because their reactivities can be controlled by varying the alloy composition.

Because many heterogeneous catalytic reactions occur at metal surfaces, much of the computational work on metal alloys has focused on surface segregation phenomena. The study of surface segregation is important, because the actual surface composition governs heterogeneous reaction rates. Of particular interest to the current work are the previous calculations using Monte Carlo methods ${ }^{3,4,5,6}$ which have attempted to predict or explain surface composition. These previous calculations have used a variety of potential models ranging from nearest-neighbor interactions $^{5,6}$ to Lennard-Jones forces. ${ }^{3,4}$ More recently, Foiles ${ }^{7}$ studied surface segregation in $\mathrm{Ni}-\mathrm{Cu}$ alloys using embedded atom potentials, and found good agreement with experimental data. In addition to previous studies on bulk alloys, work on alloy clusters was carried out by Tsai,

\footnotetext{
${ }^{a)}$ Present address: Departamento de Ciencias y Tecnologia, Universidad Interamericana, San Juan, Puerto Rico, 00919-1293.
}

Abraham, and Pound. ${ }^{8}$ Using Monte Carlo methods the structure of $\mathrm{Ar}-\mathrm{Kr}, \mathrm{Cu}-\mathrm{Ni}, \mathrm{Cu}-\mathrm{Ru}$, and $\mathrm{Cu}-\mathrm{O}$ s clusters were investigated. The intermolecular forces were modeled by Lennard-Jones interactions, and emphasis was placed on radial distribution functions and structure factors.

In the current work we present the results of calculations of the heat capacity of $\mathrm{Pd}_{6} \mathrm{Ni}_{7}$ as a function of temperature. We shall find that the heat capacity is a useful and interesting probe of the structural properties of the cluster. We have chosen to study palladium-nickel clusters because of available potential energy parameters ${ }^{9,10}$ and the importance of the particular alloy to catalytic reactions. ${ }^{11}$ In previous studies of the heat capacity of 13 atom single component clusters, anomalies have been identified that are associated with coexistence regions between solidlike and liquidlike forms. ${ }^{12,13}$ These heat capacity anomalies are associated with isomerization transitions and have been interpreted in terms of melting. ${ }^{12}$ For alloy clusters we shall find the same melting behavior. We shall also find isomerization anomalies in the heat capacity at temperatures significantly below the melting transition. These low temperature heat capacity anomalies will be found to be reminiscent of disordering transitions in bulk alloys. ${ }^{14}$ To our knowledge, this type of transition has not been observed before in alloy clusters. We shall find that there are significant quasiergodicity sampling difficulties in simulating the low temperature heat capacity. As in previous work ${ }^{13,15,16}$ the problem of quasiergodicity will be solved by J-walking.

The contents of the remainder of this paper are as follows. In the next section we outline the computational approach to the calculation including a discussion of the potential model used. We give results of simulated annealing studies of isomer structures in Sec. III, and in Sec. IV we give the results of the heat capacity calculations. Finally, in Sec. V we discuss the implications of our work. 


\section{THEORETICAL MODEL}

\section{A. Interaction potential}

In this work we have simulated the properties of nickel-palladium clusters using both the Lennard-Jones (LJ) potential and an embedded atom ${ }^{17}$ (EA) potential to model the intermolecular forces. Since the EA potential introduced significant computational overhead into the calculation, the bulk of the work was performed using the $\mathrm{LJ}$ model.

In the $\mathrm{LJ}$ model, the interparticle interaction between any two pairs of atoms, $i$ and $j$, is described by the potential

$$
V\left(r_{i j}\right)=4 \epsilon_{i j}\left[\left(\sigma_{i j} / r_{i j}\right)^{12}-\left(\sigma_{i j} / r_{i j}\right)^{6}\right],
$$

where $r_{i j}$ is the distance between atoms $i$ and $j$. The Lennard-Jones parameters, $\epsilon_{i i}$ and $\sigma_{i i}$, between like atoms have been taken from the work of Halicioglu and Pound. ${ }^{9}$ For the metals of interest in this work these parameters are, $\epsilon_{i i}=6030 \mathrm{~K}$ and $\sigma_{i i}=2.282 \AA$ for nickel and $\epsilon_{i i}=4951$ $\mathrm{K}$ and $\sigma_{i i}=2.520 \AA$ for palladium. Although, the standard Berthelot-Lorentz combining rules ${ }^{18}$ have been extensively used to describe the interaction of unlike species for the rare-gases, the interaction between unlike metal atoms is inherently more complex. To explore the sensitivity of our eventual conclusions to the strength of the interaction between unlike atoms in the cluster, we have generalized the combining rules for unlike atoms to be

$$
\begin{aligned}
& \sigma_{i j}=\frac{\left(\sigma_{i i}+\sigma_{j j}\right)}{2}, \\
& \epsilon_{i j}=\alpha \sqrt{\epsilon_{i i} \epsilon_{j j}}
\end{aligned}
$$

When $\alpha=1$, Eq. (3) becomes the standard BerthelotLorentz rule. When $\alpha$ differs from 1 the interaction between unlike pairs of atoms increases or decreases depending on the magnitude of $\alpha$. As will be seen in the next section, the structural behavior of the bimetallic clusters considered here proved to depend scnsitively on $\alpha$.

It is well known that simple pairwise potentials do not provide an accurate description of the interactions between metal atoms where many-body effects play a crucial role. Recently, Daw and Baskes ${ }^{17}$ have proposed a semiempirical method for the computation of energies of metallic systems. This interaction, called the EA potential, assumes that the energy of the system is given by two contributions; the energy necessary to embed an atom into the local electron density of the remainder atoms, and an electrostatic interaction that is represented in a pairwise fashion. These contributions are determined empirically by fitting several parameters to properties of the bulk material. This potential has been applied to a wide variety of problems including surface reconstruction studies, ${ }^{19}$ the investigation of surface segregation in alloys, ${ }^{7}$ and the study of liquids. ${ }^{20}$ More recently, Vlachos, Schmidt, and Aris ${ }^{21}$ have used this potential in the study of the structural behavior of small nickel clusters. They found that for clusters of size $n=2-7$, 9-15, and 19, no significant structural differences were observed between EA and LJ results. We have performed several exploratory calculations using this potential. As will be discussed later, our results were in general agreement with Vlachos et al. for 13 atom clusters; no qualitative differences between the structure of the $\mathrm{LJ}$ and $\mathrm{EA}$ clusters were observed.

Physical clusters in the gas phase have finite vapor pressures, and evaporation processes imply that the notion of a cluster must be defined with care. We have adopted the definition used by Lee, Barker, and $\mathrm{Abraham}^{22}$ of confining the atoms to a perfectly reflecting constraining potential of radius $R_{c}$ centered on the center-of-mass of the cluster. For all calculations performed in this work the constraining radius was set to $3 \sigma_{i i}$, where $\sigma_{i i}$ is the $\mathbf{L J}$ parameter for palladium. This radius was large enough to allow free movement of the atoms through physically significant regions of configuration space but small enough to force any evaporations to return to the cluster in time scales short compared to the total simulation time.

\section{B. Computational approach}

In the calculations that are discussed in the next section, we have investigated the configurations of the icosahedral-like isomers of the alloy clusters, and we have used Monte Carlo methods to investigate the thermodynamic properties of the clusters. In addition to their intrinsic interest, the set of icosahedral isomers is important because in standard Metropolis Monte Carlo methods, ${ }^{23,24}$ it is convenient to begin the simulations with the most stable structures. The difficulties associated with finding absolute minima for one component clusters are considerable. The difficulty is more severe in alloys because of the significantly larger number of isomers available. To obtain an understanding of the isomeric structures, a simulated annealing procedure based on Brownian dynamics was used. This same method was used in previous studies of hydrogen fluoride clusters ${ }^{25}$ and argon clusters adsorbed on graphite. ${ }^{15}$ In this approach, the motions of the particles are propagated according to the Langevin equation

$$
\frac{d \mathbf{v}_{i}}{d t}=-\gamma \mathbf{v}_{i}+\frac{1}{m_{i}} \mathbf{F}_{1}+\frac{1}{m_{i}} \mathbf{F}_{2}
$$

where $\gamma$ is a friction constant, $\mathbf{v}_{i}$ is the velocity of particle $i, m_{i}$ is the mass of particle $i, \mathbf{F}_{1}$ is the force on particle $i$ from the other particles, and $\mathbf{F}_{2}$ is a random force. The properties of $\mathbf{F}_{2}$ and the method used to solve $\mathbf{E q}$. (4) are given in Chandrasekhar's classic review of Brownian motion. ${ }^{26}$ Equation (4) was solved at an initial temperature of $4951 \mathrm{~K}$ (the well depth of the Pd-Pd interaction potential) for a warm-up period. At this temperature the system is hot enough to behave as a liquid, hence allowing for a more efficient sampling in the annealing procedure. This warm-up period was followed by instantaneous cooling to $0 \mathrm{~K}$. To increase the confidence that this procedure located the absolute minimum in the potential energy surface, from 1000 to 2000 trajectories were calculated for each cluster considered here. 
The constant volume heat capacity of clusters consisting of $n$ particles was calculated using the standard expression in terms of the energy fluctuations of the system

$$
C_{v}=\frac{\left\langle V^{2}\right\rangle-\langle V\rangle^{2}}{k_{B} T^{2}}+\frac{3 n k_{B}}{2}
$$

In Eq. (5) $V$ is the potential energy, $T$ is the temperature, and $k_{B}$ is the Boltzmann constant. Attempts were made to evaluate the canonical averages appearing in Eq. (5) with standard Metropolis Monte Carlo methods. ${ }^{23}$ However, as in single component clusters, quasiergodicity difficulties were encountered. ${ }^{13}$ To solve the quasiergodicity difficulties in the Monte Carlo simulations, we used the J-walking method. ${ }^{13}$ This approach is based on coupling the usual Metropolis random walk with periodic jumps to another random walker at a higher temperature. In the standard Metropolis algorithm, a random walker samples configuration space from an initial position $x_{i}$ to a final position $x_{f}$ with a probability of acceptance

$$
p=\min \left[1, q\left(x_{f} \mid x_{i}\right)\right] \text {, }
$$

where

$$
q\left(x_{f} \mid x_{i}\right)=\frac{S\left(x_{i} \mid x_{f}\right) \rho\left(x_{f}\right)}{S\left(x_{f} \mid x_{i}\right) \rho\left(x_{i}\right)}
$$

$\rho(x)$ is the Boltzmann distribution, and $S\left(x^{\prime} \mid x\right)$ the sampling distribution that is usually generated from uniform deviates over a finite range. ${ }^{27}$ In the $\mathrm{J}$-walking technique the sampling distribution for the jumps is the Boltzmann distribution at a higher temperature $\beta_{J}$

$$
S_{J}\left(x^{\prime} \mid x\right)=Z^{-1} \exp \left[-\beta_{J} V\left(x^{\prime}\right)\right],
$$

where $Z$ is the standard configurational integral. Using Eq. (8) $q\left(x^{\prime} \mid x\right)$ becomes

$$
q\left(x^{\prime} \mid x\right)=\exp \left\{\left(\beta_{J}-\beta\right)\left[V\left(x^{\prime}\right)-V(x)\right]\right\} .
$$

By periodically jumping to the high temperature distribution and using Eqs. (6) and (9) to insure detailed balance, ergodicity can be achieved in Monte Carlo simulations at the low temperature. As we shall discuss shortly, the J-walking algorithm proved to be valuable in the current work.

\section{SIMULATED ANNEALING RESULTS}

In this work we investigated the thermodynamic and structural properties of $\mathrm{Pd}_{6} \mathrm{Ni}_{7}$. We chose a 13 atom cluster because previous computational work ${ }^{12,13}$ on single component "magic numbered" clusters (e.g., 13) have found anomalies in the heat capacity in the coexistence region. The coexistence phenomena are caused by isomerizations between several solidlike and liquidlike structures and are similar to melting in bulk materials. As we shall see, in addition to heat capacity anomalies that arise from solidliquid coexistence phenomena, additional peaks in the heat capacity will be found at low temperatures from isomerizations to different icosahedral-like structures in $\operatorname{Pd}_{6} \mathrm{Ni}_{7}$.

To gain more understanding of the low energy isomers, simulated annealing was used to explore the potential en-

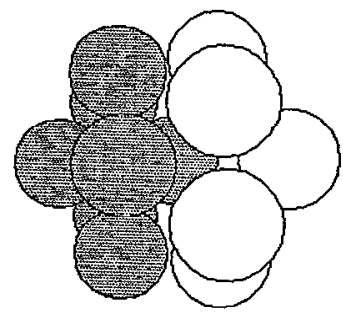

FIG. 1. The lowest energy structure of $\operatorname{Pd}_{6} \mathrm{Ni}_{7}$ for $\alpha=1.0$. In this completely segregated structure with a mixing number of 16 , the shaded atoms are palladium and the unshaded atoms are nickel.

ergy surface. To describe the interaction energy between unlike atoms, $\alpha$ was allowed to range between 0.95 and 1.1. To characterize the arrangement of atoms in a specific cluster, we defined the mixing number, $M_{N}$, to be the number of Pd-Ni nearest-neighbor interactions in a particular cluster. $M_{N}$ was computed by counting the number of distinct $\mathrm{Ni}-\mathrm{Pd}$ interatomic separations that are $<1.5 \sigma_{\mathrm{Ni}-\mathrm{Pd}}$. This distance was chosen to allow for fluctuations in the bond length around the equilibrium Pd-Ni distance. The mixing number provides a measurement of the extent of heterogeneity in the cluster. For example, in a 13 atom icosahedral cluster there are a total of 42 bonds; if the structure is completely segregated (Fig. 1) the mixing number is 16 , whereas for a completely mixed cluster $M_{N}$ is 26 (Fig. 2).

Figure 1 shows the minimum energy configuration for the $\mathrm{Pd}_{6} \mathrm{Ni}_{7}$ cluster for $\alpha=1.0$ obtained from the simulated annealing procedure. It can be seen that an icosahedral-like structure is obtained, similar to the one observed for pure $\mathrm{Ar}_{13}$ in the vapor phase. ${ }^{24,28}$ The arrangement of atoms form a completely segregated structure with a mixing number of 16. Several minima were found in the potential energy surface for this cluster, all having icosahedral-like structures, but with different mixing numbers. For example, the second lowest energy equilibrium structure is a slightly distorted icosahedron with a mixing number of 18 . The second and third columns of Table I show the total energy and mixing number for the first five isomers for $\alpha=1.0$. It can be seen that for this sequence of isomers as the total energy increases the mixing number also increases. An understanding of the relation between $M_{N}$ and energy can be attained with simple bond counting argu-

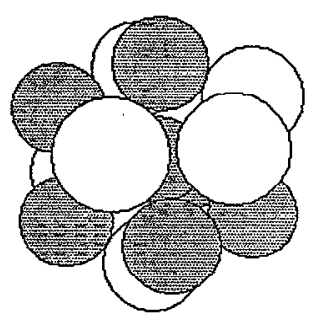

FIG. 2. The lowest energy structure of $\mathrm{Pd}_{6} \mathrm{Ni}_{7}$ for $\alpha=1.06$. In this mixed structure with a mixing number of 26 , the shaded atoms are palladium and the unshaded atoms are nickel. 
TABLE I. Total energy and mixing number for the lowest five equilibrium configurations of a $\mathrm{Pd}_{6} \mathrm{Ni}_{7}$ cluster obtained from simulated annealing using differing variables of $\alpha$.

\begin{tabular}{cccccccc}
\hline \hline & $\alpha=1.0$ & & & \multicolumn{3}{c}{$\alpha=1.06$} \\
\cline { 1 - 3 } \cline { 6 - 7 } Isomer & Energy $^{\mathrm{a}}$ & $M_{N}$ & & Isomer & Energy $^{\mathrm{a}}$ & $M_{N}$ \\
\hline 1 & -50.63118 & 16 & & 1 & -52.23346 & 26 \\
2 & -50.59303 & 18 & & 2 & -52.16241 & 24 \\
3 & -50.57654 & 18 & & 3 & -52.15231 & 24 \\
4 & -50.53860 & 20 & & 4 & -52.15406 & 24 \\
5 & -50.52191 & 22 & & 5 & -52.09509 & 22 \\
\hline \hline
\end{tabular}

"The total energy is expressed in units of $\epsilon_{\mathrm{Pd}-\mathrm{Pd}}$.

ments similar to those used elsewhere, ${ }^{24,29}$ in rationalizing the structures of pure clusters. In bond counting schemes we take the contribution of one Pd-Pd nearest-neighbor to the interaction energy to be $1.0 \epsilon_{\mathrm{Pd}-\mathrm{Pd}}$, whereas Ni-Pd and $\mathrm{Ni}-\mathrm{Ni}$ near neighbors contribute $1.104 \epsilon_{\mathrm{Pd}-\mathrm{Pd}}$ and 1.218 $\epsilon_{\mathrm{Pd}-\mathrm{Pd}}$, respectively. In the completely segregated structure there are $16 \mathrm{Ni}-\mathrm{Ni}$ near neighbors, $16 \mathrm{Pd}-\mathrm{Ni}$ ncar neighbors, and $10 \mathrm{Pd}-\mathrm{Pd}$ near neighbors, giving an interaction energy of $47.152 \epsilon_{\mathrm{Pd}-\mathbf{P d}_{d}}$. On the other hand, the second lowest equilibrium structure, having a mixing number of 18, has $15 \mathrm{Ni}-\mathrm{Ni}$ bonds, $18 \mathrm{Ni}-\mathrm{Pd}$ bonds, and $9 \mathrm{Pd}-\mathrm{Pd}$ bonds, for an interaction energy of $47.142 \epsilon_{\mathrm{Pd}_{\mathrm{d}} \mathrm{Pd}}$. By simply combining the interaction energy contribution from nearest neighbors one obtains the same qualitative results as the detailed calculation. It is important to notice that the difference in energy between the first five lowest energy structures is small. For example, the difference between the first and the second lowest energy structure is $189 \mathrm{~K}$. This small difference in energy suggests that at low temperatures several icosahedral-like isomers, only differing in mixing number, should be accessible by the system.

Figure 2 shows the minimum energy configuration for the $\mathrm{Pd}_{6} \mathrm{Ni}_{7}$ cluster for $\alpha=1.1$. As in the previous case, the lowest energy configuration has an icosahedral-like structure. However, in contrast to the case that $\alpha=1.0$, this cluster has a completely mixed structure, i.e., mixing number 26. Columns five and six of Table I show the energy and mixing number for the first five lowest isomers for $\alpha=1.1$. For $\alpha=1.1$ the energy gap between the different isomers is larger than for $\alpha=1.0$. Accessing these low lying isomers at low temperature will be less probable. As opposed to the behavior observed for $\alpha=1.0$, as the mixing number decreases the energy of the bimetallic alloy increases. Interestingly, these results show a strong dependence between the mixing number and the value of $\alpha$. By changing $\alpha$ by $10 \%$ a significant change in the qualitative structural nature of the cluster alloy is observed.

To understand in more detail the dependence of structure on $\alpha$, additional annealing studies were performed using several $\alpha$ values ranging from 0.95 to 1.2. Figure 3 is a graph of the mixing number of the lowest energy configuration obtained from simulated annealing as a function of $\alpha$. For values of $\alpha$ smaller than 1.0 the mixing number of the lowest energy configuration is always 16 , whereas for values of $\alpha$ greater than 1.0225 the mixing number of the

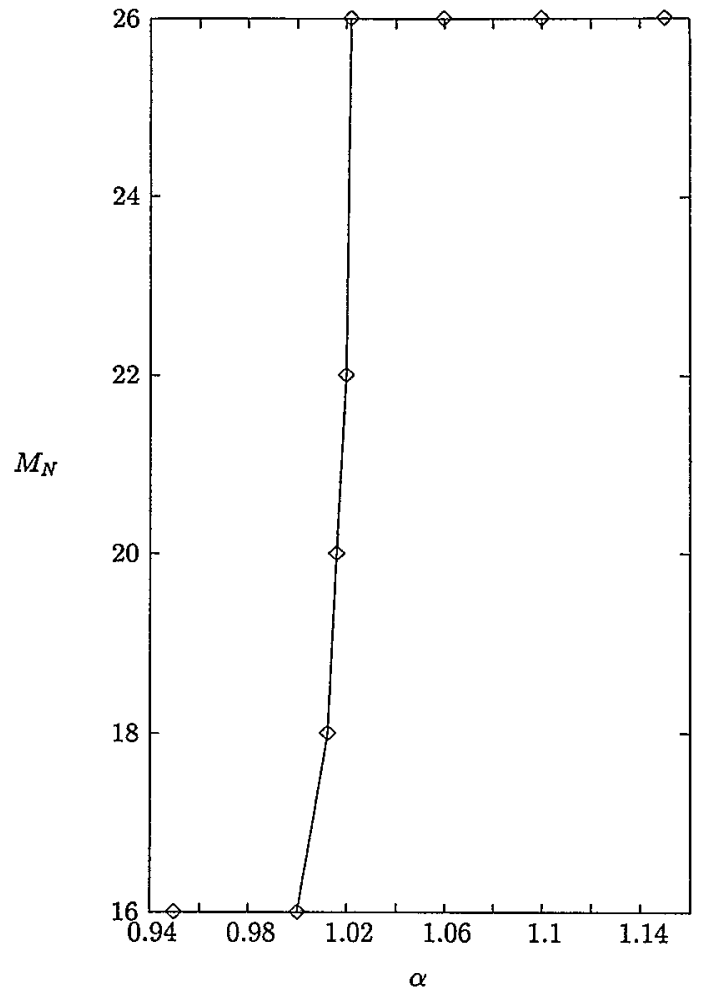

FIG. 3. The mixing number $M_{N}$ of the lowest energy structure of $\operatorname{Pd}_{6} \mathrm{Ni}_{7}$ as a function of $\alpha$. The rapid change in $M_{N}$ with $\alpha$ is apparent.

lowest energy configuration is always 26 . Values of $\alpha$ between 1.0 and 1.0225 show intermediate mixing numbers for the lowest energy structure. The transition between completely segregated structures and mixed structures as a function of $\alpha$ is remarkably steep.

Simulated annealing studies were also performed using the EA potential. The results obtained were in qualitative agreement with the $\mathrm{LJ}$ results obtained for $\alpha=1.06$. The lowest equilibrium structure found when the EA potential was used was a slightly distorted icosahedron with a mixing number of 26. Examination of the potential curve for $\mathrm{Pd}-\mathrm{Ni}$ using the EA potential showed that this potential is similar to the simple $\mathrm{LJ}$ potential with $\alpha=1.06$. Moreover, examination of three-body effects using the EA potential seems to suggest a small contribution to the energy. To analyze the contribution of three-body effects we examined how the interaction energy between two atoms was modified by the presence of a third atom. As the third atom approached the other two in a direction perpendicular to the diatomic bond, we observed that the energy varied by only $10 \%$. This same variation was found for pure metals as well. Consequently, the qualitative nature of the thermodynamic properties obtained using either the $\mathbf{L J}$ or the EA potentials can be expected to be the same. Because the computational overhead is high when the EA potential is used, we used the $\mathrm{LJ}$ potential with $\alpha=1.0$ and 1.06 in the Monte Carlo simulations that follow. 


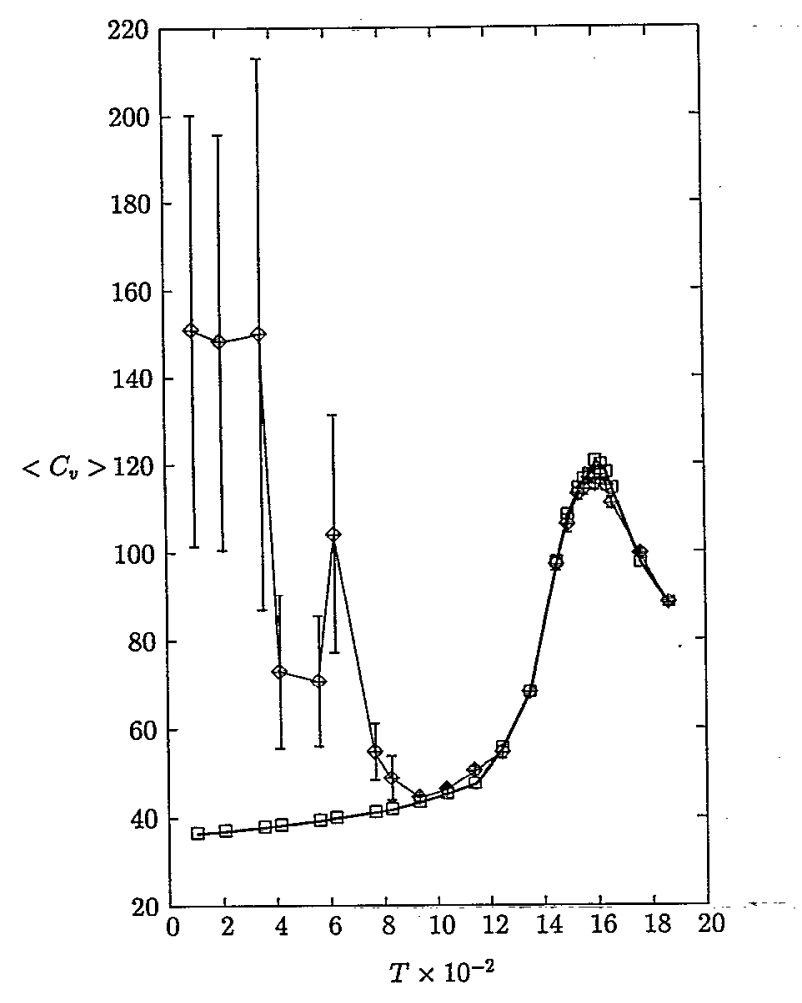

FIG. 4. The constant volume heat capacity of $\mathrm{Pd}_{6} \mathrm{Ni}_{7}$ with $\alpha=1.0$ as a function of temperature calculated using Metropolis sampling. The curve connecting the points represented by squares was generated using the lowest energy structure as the initial configuration and the curve connecting the points represented by diamonds used random initialization. The large error bars at low temperatures with random initialization is a result of quasiergodicity difficulties.

\section{MONTE CARLO CALCULATION OF THE HEAT CAPACITIES}

The heat capacity at each temperature was calculated from 100 independent initialized walks, each consisting of $10^{5}$ warm-up moves followed by $10^{5}$ moves where data were accumulated. Two different starting configurations were used as input for the Monte Carlo simulation; the lowest energy equilibrium configurations at $0 \mathrm{~K}$, and a randomly initialized cluster. The particles were moved using a Metropolis box size such that $50 \%$ of the moves wcre accepted.

Figure 4 shows the heat capacity as a function of temperature for the $\mathrm{Pd}_{6} \mathrm{Ni}_{7}$ cluster for $\alpha=1.0$. The lower curve (connecting points represented by squares) corresponds to initialization from the lowest equilibrium structure and the upper curve (connecting points represented by diamonds) corresponds to a random initialization. It can be observed that in both cases a coexistence region similar to the one observed for pure argon ${ }^{12,13}$ is obtaincd. The maximum in the coexistence region occurs at $1600 \mathrm{~K}$. However, at low temperatures, different behavior between the two initializations is observed. The lower curve shows no apparent anomalies in the heat capacity; the usual monotonic decrease with increasing temperature is observed. The randomly initialized curve shows large values and fluctuations of the heat capacity in the low temperature regime. It is clear in the former case that improper sampling of configuration space severely affects the computation of the heat capacity. From the simulated annealing results it is expected that at low temperature several isomers should be accessed. For initialization from the lowest energy configuration it was found that the average mixing number was 16. Hence, even starting with the lowest equilibrium geometry at $0 \mathrm{~K}$, quasiergodicity difficulties can be expected.

To eliminate quasiergodicity problems we have used the J-walking method introduced by Frantz et al. ${ }^{13}$ In this approach the usual Metropolis random walker is coupled with another walker at a higher temperature to which periodic jumps are attempted. The J-walker distributions were generated in stages, just as in the previous work using the J-walking method. An initial distribution was generated at $T=2073 \mathrm{~K}$ from a long run consisting of $1 \times 10^{6}$ warm-up moves followed by $5 \times 10^{7}$ moves where a configuration was stored every 1000 moves. The high temperature and the large number of steps used to generate the $\mathrm{J}$-walking distribution ensure full sampling of configuration space, hence reducing quasiergodicity. This distribution was used to generate additional $\mathbf{J}$-walking distributions over the temperature range of $207<T<2073$ with a mesh size of $\Delta T=207 \mathrm{~K}$. For example, the $1866 \mathrm{~K}$ distribution was generated from $5 \times 10^{5}$ warm-up moves followed by $5 \times 10^{6}$ moves with a configuration stored every 100 moves and jumps attempted to the $2073 \mathrm{~K}$ distribution every 10 moves. This resultant distribution was used to generate the $1659 \mathrm{~K}$ distribution, and so on. The distributions were generated and saved in an external array. For the range of $20<T<207 \mathrm{~K}$ a step size of $\Delta=20 \mathrm{~K}$ was used in order to obtain appreciable overlap between the low temperature and high temperature walkers. This dichotomy between two regimes of temperature will be discussed below. For the J-walking temperatures used in this study, from $20 \%$ to $40 \%$ of the attempted jumps were accepted depending upon the particular temperature range.

Figure 5 shows the heat capacity as a function of temperature for the $\mathrm{Pd}-\mathrm{Ni}$ bimetallic alloy with $\alpha=1.0$. The lower curve (connecting points represented by pluses) represents initialization from the lowest energy configuration using standard Metropolis sampling and the upper curve (connecting points represented by diamonds) represents random initialization with $\mathrm{J}$-walking. It can be observed that in the melting region, the behavior of both curves are similar, although the J-walking generated curve is smoother. Examination of the standard deviation in the heat capacities as a function of temperature (Fig. 6) shows a decrease in the fluctuations in the melting region when $\mathbf{J}$-walking is used. This behavior is similar to that observed by Frantz et al. ${ }^{13}$ for an $\mathrm{Ar}_{13}$ cluster. As previously stated, at low temperatures the standard Monte Carlo results show no anomalous behavior in the heat capacity. However, the J-walking curve shows a small maximum in the heat capacity in the region of $42-207 \mathrm{~K}$. As in the melting region this anomaly in the heat capacity is caused by the accessibility of high energy isomers in that temperature regime. 


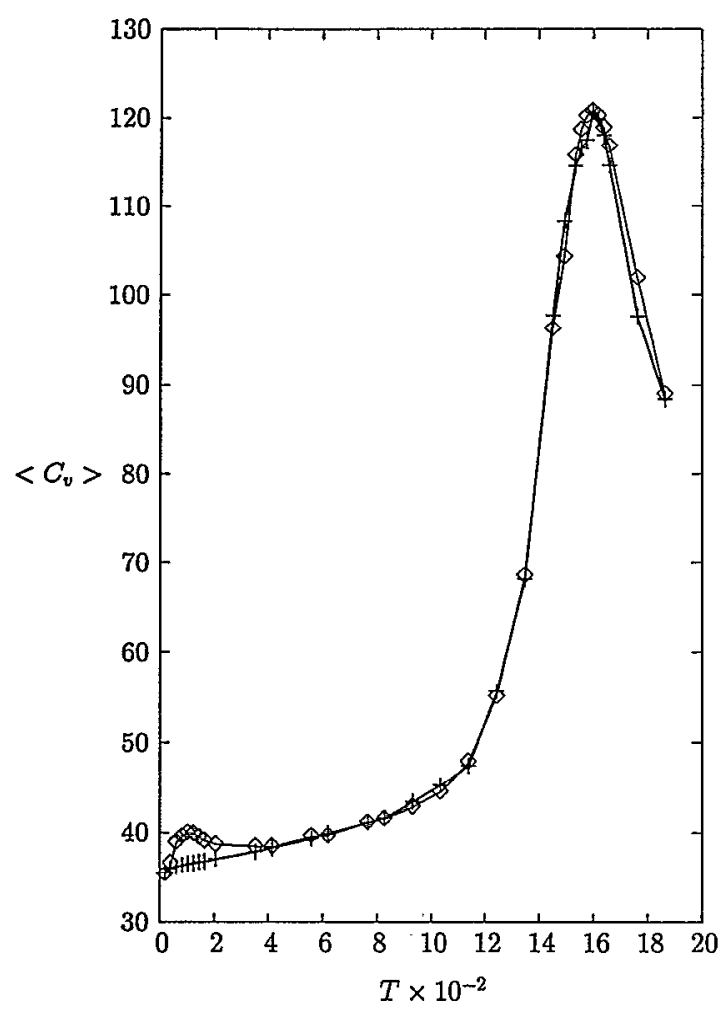

FIG. 5. The constant volume heat capacity of $\mathrm{Pd}_{6}-\mathrm{Ni}_{7}$ with $\alpha=1.0$ as a function of temperature. The curve connecting points represented by pluses was generated using Metropolis sampling with the lowest energy structure as the initial configuration and the curve connecting points represented by diamonds was generated using J-walking with random initializations. The melting anomaly is evident by the maximum at 1600 $\mathrm{K}$ and the mixing anomaly is evident by the maximum at $75 \mathrm{~K}$.

To characterize this low temperature isomerization phenomenon in more detail, the fraction of isomers with a given mixing number was calculated. For this purpose we have defined $X_{1}$ to be the fraction of configurations in the simulation at temperature $T$ having mixing numbers associated with the lowest energy structure. For $\alpha=1.0, X_{1}$ corresponds to the fraction of configurations with mixing number equal to 16 . For $\alpha=1.06, X_{1}$ corresponds to the fraction of configurations with mixing number equal to 26 . As stated before $X_{1}$ was equal to 1.00 for all temperatures below $415 \mathrm{~K}$ using standard Monte Carlo and initialization from the lowest equilibrium structure. Figure 7 shows $X_{1}$ as a function of temperature for two different values of $\alpha$. The data were accumulated using the J-walking Monte Carlo technique in the same manner as in the computation of the heat capacity as described above. For $\alpha=1.0$ (lower curve with points represented by diamonds), it is observed that only at low temperature is $X_{1}=1.0$. At a temperature of $104 \mathrm{~K}$ other isomers are accessed, and $X_{1}$ decreases. In the same range of temperatures where the anomalies in the heat capacities are observed, a rapid drop in $X_{1}$ occurs. Accessibility of high energy isomers with different mixing numbers at low temperatures causes the anomaly in the heat capacity.

As stated previously, the temperature step size that was used to generate the J-walking configuration in the

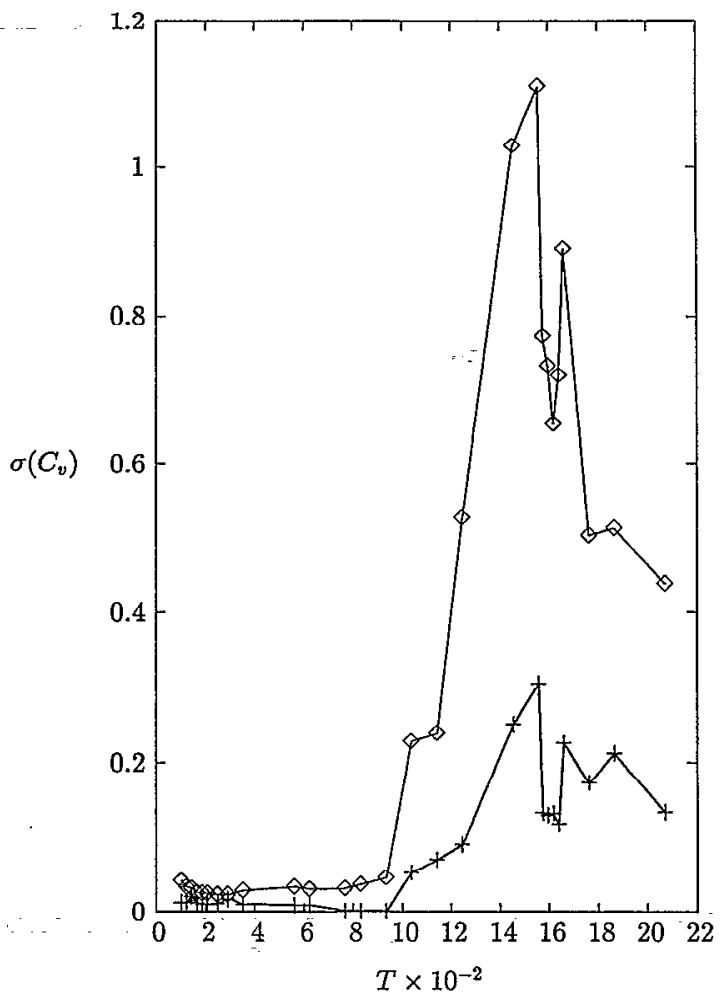

FIG. 6. The standard deviation of the heat capacity as a function of temperature. The curve connecting the points represented by diamonds was generated with Metropolis sampling using the lowest energy structure as the initial configuration and the curve connecting the points represented by pluses was generated with $\mathbf{J}$-walking using random initializations. The significant improvement in the fluctuations of the heat capacity from J-walking is evident.

region where the low temperature anomaly was observed, had to be reduced by about a factor of 10 to attain acceptable overlap between the low temperature and the high temperature walker. If the step size was not reduced the acceptance probability was nearly zero. The overlap between the configurations in the $\mathbf{J}$-walking algorithm can provide information related to physical processes that might be occurring in certain temperature regimes.

Figure 8 shows the heat capacity as a function of temperature for $\alpha=1.06$. As in Fig. 5, the curve connecting the points represented by diamonds is the Metropolis result from an initialization to the lowest energy structure and the curve connecting the points represented by pluses is the $\mathrm{J}$-walking result generated with random initializations. For $\alpha=1.06$ the difference in energy between the different isomers is greater than when $\alpha=1.0$. At low temperatures for $\alpha=1.06$ we observe a heat capacity anomaly occurring at a higher temperature but with a smaller maximum that for $\alpha=1.0$. In examining the upper curve in Fig. 7 (with calculated points represented by pluses), it can be noted that although higher energy isomers are being accessed at low temperature, the fraction in the ground state is larger than when $\alpha=1.0$ and $X_{1}$ decreases almost linearly with increasing temperature. Consequently the low temperature anomaly in Fig. 8 is flatter and shifted to a higher temperature than the low temperature anomaly depicted in Fig. 5. 


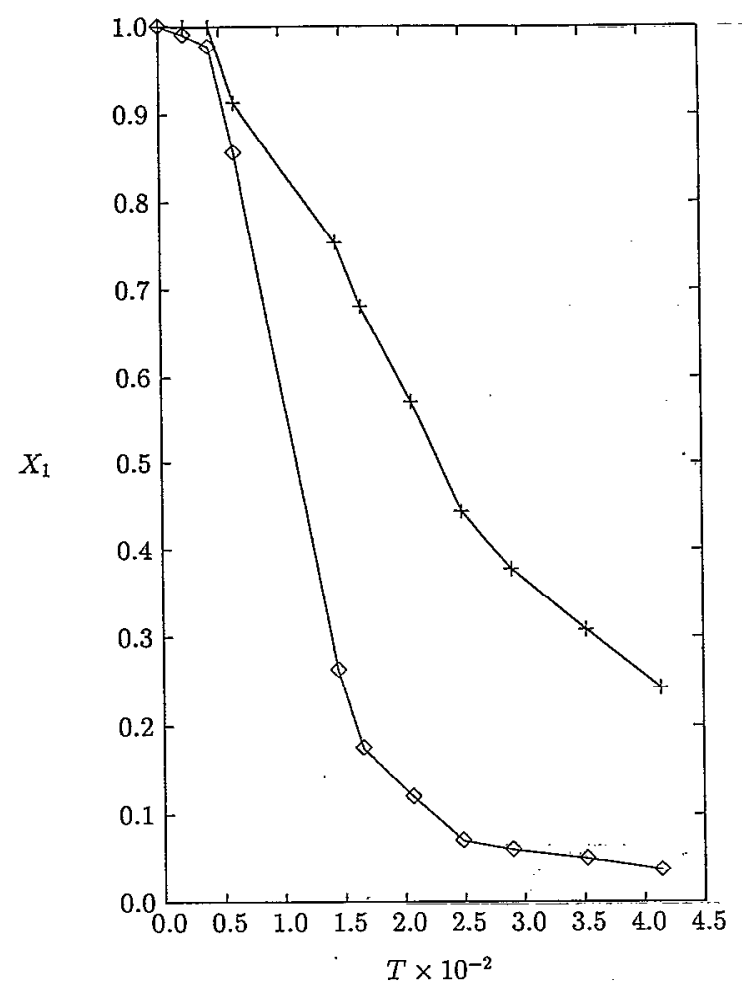

FIG. 7. The fraction of configurations having mixing numbers associated with the ground state isomer as a function of temperature. The curve connecting points represented by diamonds is for $\alpha=1.0$ and the curve connecting points represented by pluses is for $\alpha=1.06$. The rapid decrease in $X_{1}$ with temperature for $\alpha=1.0$ matches the low temperature heat capacity anomaly in Fig. 5.

For $\alpha=1.06$ there is not a rapid drop in $X_{1}$ with temperature. The more gradual decrease for $\alpha=1.06$ is caused by the larger gap between the lowest energy isomer and the higher energy isomers.

\section{v. CONCLUSION}

We have demonstrated the existence of low temperature isomerization transitions in alloy clusters where the structural integrity of the cluster is maintained but the connectivities of the constituent atoms are changed. These isomerization transitions are reminiscent of order-disorder transitions observed in some bulk alloy materials. ${ }^{14}$ It would be of interest to examine the heat capacity of palladium-nickcl alloy clusters as a function of cluster size to determine whether the low temperature anomaly observed here would persist. Furthermore a study of the heat capacity of a $50 \%$ mol mixture of palladium-nickel alloy in the bulk using the same potential model used in this work would show whether a disordering transition occurs in bulk phases of this system. Work on these issues are in progress.

The entropy change $\Delta S_{t}$ associated with the low temperature isomerization transition can be calculated from the data given in Fig. 5 using the expression

$$
\Delta S_{t}=\int_{0}^{T_{1}} \frac{\Delta C_{v} d T}{T}
$$

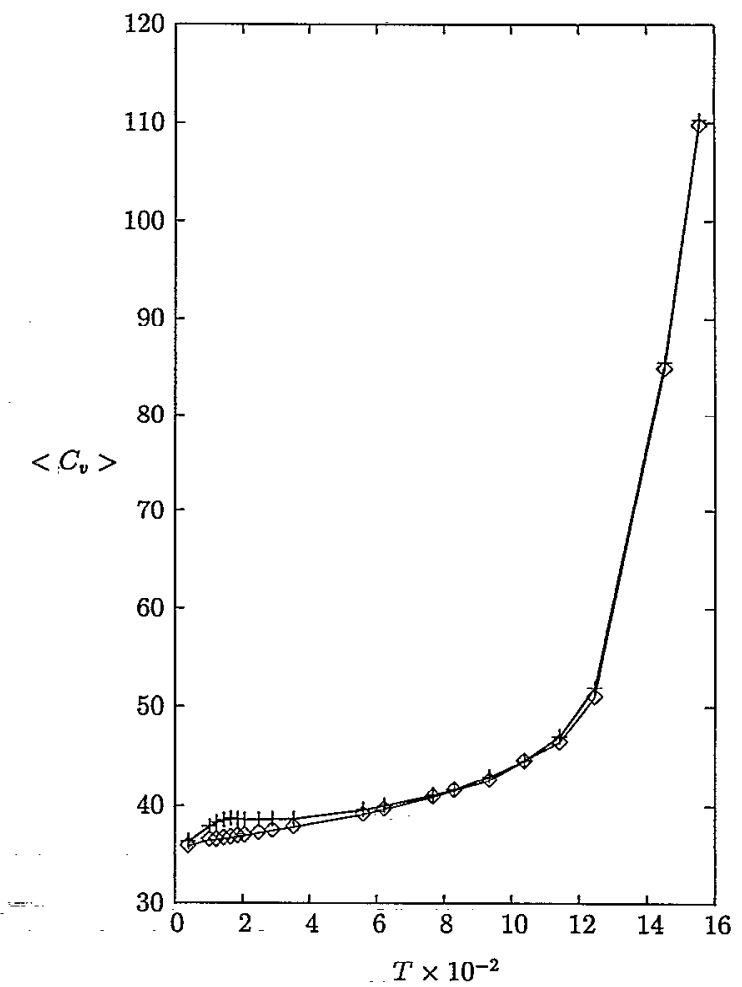

FIG. 8. The constant volume heat capacity of $\mathrm{Pd}_{6} \mathrm{Ni}_{7}$ with $\alpha=1.06$ as a function of temperature. The curve connecting the points represented by diamonds was generated using Metropolis sampling with the lowest energy structure as the initial configuration and the curve connecting points represented by pluses was generated using $J$-walking with random initializations.

where $T_{1}$ is a temperature just above the low temperature anomaly and $\Delta C_{v}$ is the difference in the heat capacity calculated with and without $J$-walking. The values of entropy changes so calculated in other ordering transitions can be used to generate physical models useful in the interpretation of a transition. ${ }^{14}$ Using trapezoid quadrature, in our case we find $\Delta S_{t} / k_{B}=4.8$ for the case that $\alpha=1$ and $\Delta S_{t} / k_{B}=2.9$ for the case that $\alpha=1.06$. Because it was not practical to locate all icosahedral-like structures with the Brownian dynamics method used in this work, we did not have sufficient data to explain the calculated values of the entropy change for the transition with a simple model. However, the magnitude of $\Delta S_{t}$ is a measure of the significant number of isomers accessed by the $\mathrm{J}$-walking calculation in the transition region.

It is important to recognize that the results reported in this work are computations based on a simple potential model of nickel-palladium alloy clusters. The intermolecular forces in the real physical system can be expected to be much more complex, and we make no prediction of any heat capacity anomalies that might be present in the real system. The significant sensitivity of the low energy structures to $\alpha$ suggests that the physical system may behave differently than the calculations predict. However, low temperature heat capacity anomalies similar to those found here can be expected to occur in a variety of physical systems. The current calculations propose the kinds of cir- 
cumstances that the anomalies might be found to occur. Continuing investigations seeking heat capacity anomalies in other physical systems are in progress.

\section{ACKNOWLEDGMENTS}

We would like to thank Dr. Steve Rick, Professor Don Frantz, and Professor Jim Doll for helpful discussions about this work. We also thank Dr. Rick for providing the code used in the EA calculations. Acknowledgment is made to the Donors of the Petroleum Research Fund of the American Chemical Society for support of this research.

${ }^{1}$ F. F. Abraham, Homogeneous Nucleation Theory (Academic, New York, 1974).

${ }^{2}$ R. D. Gonzalez, Appl. Surf. Sci. 19, 181 (1984).

${ }^{3}$ N. H. Tsai, F. F. Abraham, and G. M. Pound, Surf. Sci. 77, 465 (1978).

${ }^{4}$ F. F. Abraham, N. H. Tsai, and G. M. Pound, Surf. Sci. 78, 181 (1978).

${ }^{5}$ V. S. Sundaram and P. Wynblatt, Surf. Sci. 52, 569 (1975).

${ }^{6}$ R. G. Donnelly and T. S. King, Surf. Sci. 74, 89 (1978).

${ }^{7}$ S. M. Foiles, Phys. Rev. B 32, 7685 (1985).

${ }^{8}$ N. H. Tsai, F. F. Abraham, and G. M. Pound, Surf. Sci. 77, 465 (1978).

${ }^{9}$ T. Halicioglu and G. M. Pound, Phys. Status Solidi 30, 619 (1975).

${ }^{10}$ S. M. Foiles, M. I. Baskes, and M. S. Daw, Phys. Rev. B 33, 7983 (1986); 37, 10378 (1988).

${ }^{11}$ V. Mintsa-Eya, L. Hilaire, F. G. Gault, B. Moraweck, and A. Renouprez, J. Catal. 76, 169 (1982).

${ }^{12}$ R. H. Berry, T. L. Beck, and H. L. Davis, in Advances in Chemical Physics, edited by I. Prigogine and S. A. Rice (Wiley, New York, 1988), Vol. $70 B$, p. 75 .
${ }^{13}$ D. D. Frantz, D. L. Freeman, and J. D. Doll, J. Chem. Phys. 93, 2769 (1990).

${ }^{14}$ N. G. Parsonage and L. A. K. Staveley, Disorder in Crystals (Clarendon, Oxford, 1978).

${ }^{15}$ M. A. Strozak, G. E. Lopez, and D. L. Freeman, J. Chem. Phys. 97, 4445 (1992).

${ }^{16}$ C. J. Tsai and K. D. Jordan, J. Chem. Phys. 95, 3850 (1991).

${ }^{17}$ M. S. Daw and M. I. Baskes, Phys. Rev. Lett. 50, 1285 (1983); Phys. Rev. B 29, 6443 (1984).

${ }^{18}$ R. A. Aziz, in Inert Gases, edited by M. L. Klein (Springer, Berlin, 1984), p. 5.

${ }^{19}$ M. S. Daw and S. M. Foiles, Phys. Rev. Lett. 59, 2756 (1987); B. W. Dobson, Phys. Rev. B 35, 880 (1987).

${ }^{20}$ S. M. Foiles, Phys. Rev. B 32, 3409 (1985).

${ }^{21}$ D. G. Vlachos, L. D. Schmidt, and R. Aris, J. Chem. Phys. 96, 6880, 6891 (1992).

${ }^{22}$ J. K. Lee, J. A. Barker, and F. F. Abraham, J. Chem. Phys. 58, 3166 (1973).

${ }^{23}$ N. Metropolis, A. W. Rosenbluth, M. H. Rosenbluth, A. H. Teller, and E. Teller, J. Chem. Phys. 21, 1087 (1953).

${ }^{24}$ D. L. Freeman and J. D. Doll, in Advances in Chemical Physics, edited by I. Prigogine and S. A. Rice (Wiley, New York, 1988), Vol. 70B, p. 139.

${ }^{25}$ C. Zhang, D. L. Freeman, and J. D. Doll, J. Chem. Phys. 91, 2489 (1989).

${ }^{26}$ S. Chandrasekhar, Rev. Mod. Phys. 15, 1 (1943).

${ }^{27}$ M. H. Kalos and P. A. Whitlock, Monte Carlo Methods (Wiley, New York, 1986).

${ }^{28}$ M. R. Hoare and P. Pal, Adv. Phys. 20, 161 (1971).

${ }^{29}$ J. A. Northby, J. Chem. Phys. 87, 6166 (1987). 
The Journal of Chemical Physics is copyrighted by the American Institute of Physics (AIP). Redistribution of journal material is subject to the AIP online journal license and/or AIP copyright. For more information, see http:/ojps.aip.org/jcpo/jcpcr/jsp Copyright of Journal of Chemical Physics is the property of American Institute of Physics and its content may not be copied or emailed to multiple sites or posted to a listserv without the copyright holder's express written permission. However, users may print, download, or email articles for individual use. 
The Journal of Chemical Physics is copyrighted by the American Institute of Physics (AIP). Redistribution of journal material is subject to the AIP online journal license and/or AIP copyright. For more information, see http://ojps.aip.org/jcpo/jcper/jsp 